\title{
Citalopram and group psychotherapy in breast cancer patients: A randomized clinical trial
}

\author{
Novin Nikbakhsh ${ }^{1}$, Sussan Moudi*2, Sara Alvarzandi ${ }^{3}$, Maede Niazifar $^{4}$, Nazila Farnoush ${ }^{5}$, Ali Bijani ${ }^{6}$, \\ Marjan Moudi ${ }^{7}$
}

\begin{abstract}
Background: Depression is a common psychiatric disorder in breast cancer patients. This study was designed to evaluate the clinical efficacy of group psychotherapy on breast cancer patients with depressive disorder who took citalopram.

Methods: This clinical trial was conducted on 40 breast cancer patients with depressive disorder. The control group received citalopram $20-40 \mathrm{mg} /$ day for 12 weeks and the intervention group participated in 8 sessions of group psychotherapy in addition to the same dose of citalopram. At the baseline and 3,6, and 12 weeks after treatment, patients were followed- up. Treatment outcomes and quality of life were compared between the 2 groups.

Results: Overall, the depression score of Hospital Anxiety and Depression Scale (HADS) at baseline with the mean of 11.6 \pm 1.6 was signed in the range of clinical depression and after intervention it declined to 8.8 \pm 3.6 (in the 3rd week), 7.1 \pm 3.9 (6th week), and $5.9 \pm 4.5$ (12th week). Furthermore, HADS anxiety score at baseline with the mean of 12.6 \pm 2.6 was signed in the range of clinical anxiety and after intervention it declined to $9.1 \pm 3.0,7.3 \pm 4.1$, and $6.0 \pm 4.0$, respectively. This improvement was significantly more in the combined therapy intervention group $(\mathrm{p}<0.001)$. The mean score of quality of life based on WHO QOL-BREF questionnaire increased by 1.85 fold in the case group, improved from 44.09 to 81.70 , while the slight change was observed in the control group ( $<<0.001$ ). During the treatment, no significant adverse drug event was observed in the 2 groups $(\mathrm{p}>0.05)$.

Conclusion: Group psychotherapy has a significant effect on improving depression, anxiety, and quality of life in breast cancer patients.
\end{abstract}

Keywords: Breast cancer, Depression, Anxiety, Citalopram, Group psychotherapy

Copyright $₫$ Iran University of Medical Sciences

Cite this article as: Nikbakhsh N, Moudi S, Alvarzandi S, Niazifar M, Farnoush N, Bijani A, Moudi M. Citalopram and group psychotherapy in breast cancer patients: A randomized clinical trial. Med J Islam Repub Iran. 2018 (8 Aug);32:68. https://doi.org/10.14196/mjiri.32.68

\section{Introduction}

Breast cancer is the most prevalent invasive cancer of women worldwide, and depression is a common psychiatric disorder in these patients (1). It has been reported that $10 \%$ to $25 \%$ of breast cancer patients experience depression at any phase of their life (2), which can have a negative impact on their quality of life (3) and physical condition as well as on disease outcomes $(4,5)$. Therefore, early recognition and management of depression are important in improving quality of life and reducing distress $(1,5,6)$. There are different approaches for the management of depressive dis order in cancer patients such as psychoso-

Corresponding author: Dr Sussan Moudi, sussan.mouodi@gmail.com

1. Cancer Research Center, Babol University of Medical Sciences, Babol, Iran.

2. Social Determinants of Health Research Center, Health Research Institute, Babol University of Medical Sciences, Babol, Iran.

3. Faculty of Medicine, Babol University of Medical Sciences, Babol, Iran.

4. Department of Clinical Psychology, University of Social Welfare and Rehabilitation Sciences, Tehran, Iran.

5. Department of Surgery, Babol University of Medical Sciences, Babol, Iran.

6. SDH Research Center, Babol University of Medical Sciences, Babol, Iran.

7. Department of Internal Medicine, Babol University of Medical Sciences, Babol, Iran. cial or pharmacological interventions or combined treatment (7-9). Some previous studies have represented citalopram, a selective serotonin reuptake inhibitor (SSRI), and group psychotherapy as safe and effective interventions for the treatment of depression in breast cancer patients (7-14). However, there are a few pieces of evidence which show the superiority of any one specific intervention (either pharmacological approach or psychotherapy), and further research is necessary for different communities to define the most appropriate approach to depression when it occurs in comorbidity with breast cancer (15).

$\uparrow$ What is "already known" in this topic:

Depression is a common psychiatric problem in breast cancer patients, which can have a negative impact on physical condition, quality of life, and disease outcomes of these patients.

$\rightarrow$ What this article adds:

This study showed that combined treatment with citalopram and group psychotherapy had a significant clinical impact on depression, anxiety, and quality of life in breast cancer patients without serious physical adverse reactions. 
This study was performed to evaluate the efficacy of citalopram and group psychotherapy in breast cancer patients with depressive disorder.

\section{Methods}

\section{Design and setting of the study}

This randomized clinical trial was conducted in Babol, Northern Iran.

\section{Participants}

In the present study, the population of new cases of breast cancer patients in the stage III of the disease referred to governmental treatment centers affiliated to Babol University of Medical Sciences (which are presented as the biggest referral cancer treatment centers in northern Iran). The sample size in each group was calculated as 16 using the formula in interventional studies when the variables are quantitative, with $95 \%$ confidence interval, $80 \%$ study power, and the assumption that $\delta_{1=} \delta_{2}=3$ in HADS scores for finding 3 units of difference in HADS scores between the 2 groups after the intervention. Adding probable $20 \%$ loss to follow-up, the sample size was calculated as 20 in each group. The patients undergoing recent mastectomy surgery were invited to participate in the study. After initial counseling about the objectives of the study (performed by their surgeon), the patients were referred to a psychiatrist and examined using a clinical structured interview. A total of 40 patients diagnosed with depression according to DSM-IV TR were enrolled in the research when they met the inclusion and exclusion criteria and informed consent could be obtained.

These eligible patients were distributed in 2 groups with simple random allocation based on even or odd numbers: intervention $(n=20)$ and control group $(n=20)$.

\section{Inclusion and exclusion criteria \\ Inclusion criteria:}

1- New cases of breast cancer patients

2- Having undergone recent mastectomy surgery

3- Diagnosis of depressive disorder: mixed depression and anxiety disorder, adjustment disorder, with mixed depressive and anxiety mood, and/ or adjustment disorder with depressive mood according to DSM-IV TR

Exclusion criteria:

1- Other major psychiatric disorders, such as bipolar mood disorder, psychotic disorder, or substance use disorder

2- Mental retardation

3- Endocrine disorders

\section{Procedures and variable assessment}

At first, all participants were examined by a psychiatrist. Anxiety and depression were evaluated with a self-report rating scale of 14-items Hospital Anxiety and Depression Scale (HADS). HADS is a useful, acceptable, valid, and reliable tool for screening anxiety and depression in clinical settings $(16,17)$. This questionnaire has 2 subscales for anxiety ( 7 items) and for depression ( 7 items). For each item, the participants were asked to indicate which of the 4 options (rated from 3 to 0 ) comes closest to describ- ing how they have been feeling in the past week. The score of 0-7 indicates no clinical symptoms of anxiety or depression, 8-10 represents mild anxiety or depression, and 11-21 indicates symptomatic anxiety or depression $(16,17)$. HADS is a self-report questionnaire; however, if the patient cannot complete it, the researcher will fill out the questionnaire according to the patient's answers. The internal consistency of the Iranian version of the HADS has been found to be 0.78 for the anxiety subscale and 0.86 for the depression subscale, and its validity has been found to be 0.92 ; indicating satisfactory psychometric properties in Iranian population (18).

The patients' quality of life was assessed with the standardized scale of the Iranian version of WHO QOLBREF, whose validity, reliability, internal consistency, and dimensional structure were evaluated, and the results showed acceptable properties (19). This scale has 26 items for assessment of QOL: 2 items related to general health and overall QOL status and 24 items to evaluate its subdomains (physical and mental health, patient's dependence to the others, social relationship, environment and religious beliefs). It has been presented as a proper measure for assessing quality of life in adult Iranian populations $(19,20)$.

The control group received 20-40 mg/day citalopram (adjusted according to the therapeutic response and patient's tolerance) for 12 weeks and the intervention group received supportive group psychotherapy plus citalopram. An expert psychologist held these group psychotherapy classes. Psychotherapy classes were conducted by an expert clinical psychologist; these sessions were free, offered weekly for 8 weeks, and for at least 45 minutes in each session. All patients were reminded via phone call and text message a day before the planned session to participate in a psychotherapy class or to be visited by a physician.

The contents of these group psychotherapy classes were scheduled to help the patient to better adapt to the environment. Considering that the participants were undergoing coincidental chemotherapy, the side-effects of chemotherapy and how to interfere with these complications were discussed in each session. These 8 sessions were conducted as follow: (1) introduction of the trainer and group members to each other; stating the regulations of the group such as privacy and confidentiality; introduction of the therapeutic approach; the overall objectives of the group; the required processes to attain the objectives; the biopsychosocial dimensions of depressive disorders and emphasis to do activities which should be performed at home; (2) training the skill of problem-solving; the association between daily activities and mood; and behavioral approaches that are helpful to elevate the mood; (3) an overview of the previous class content; combining cheerful and well-behaved activities to validate equilibrium and positive reinforcement and use of past samples to demonstrate these activities; (4) an overview of the previous class content; evaluation of the outcomes and consequences of behavioral improvements; training the skill of deep abdominal breathing and doing it at the beginning of each session along with imagination; (5) an overview of the 


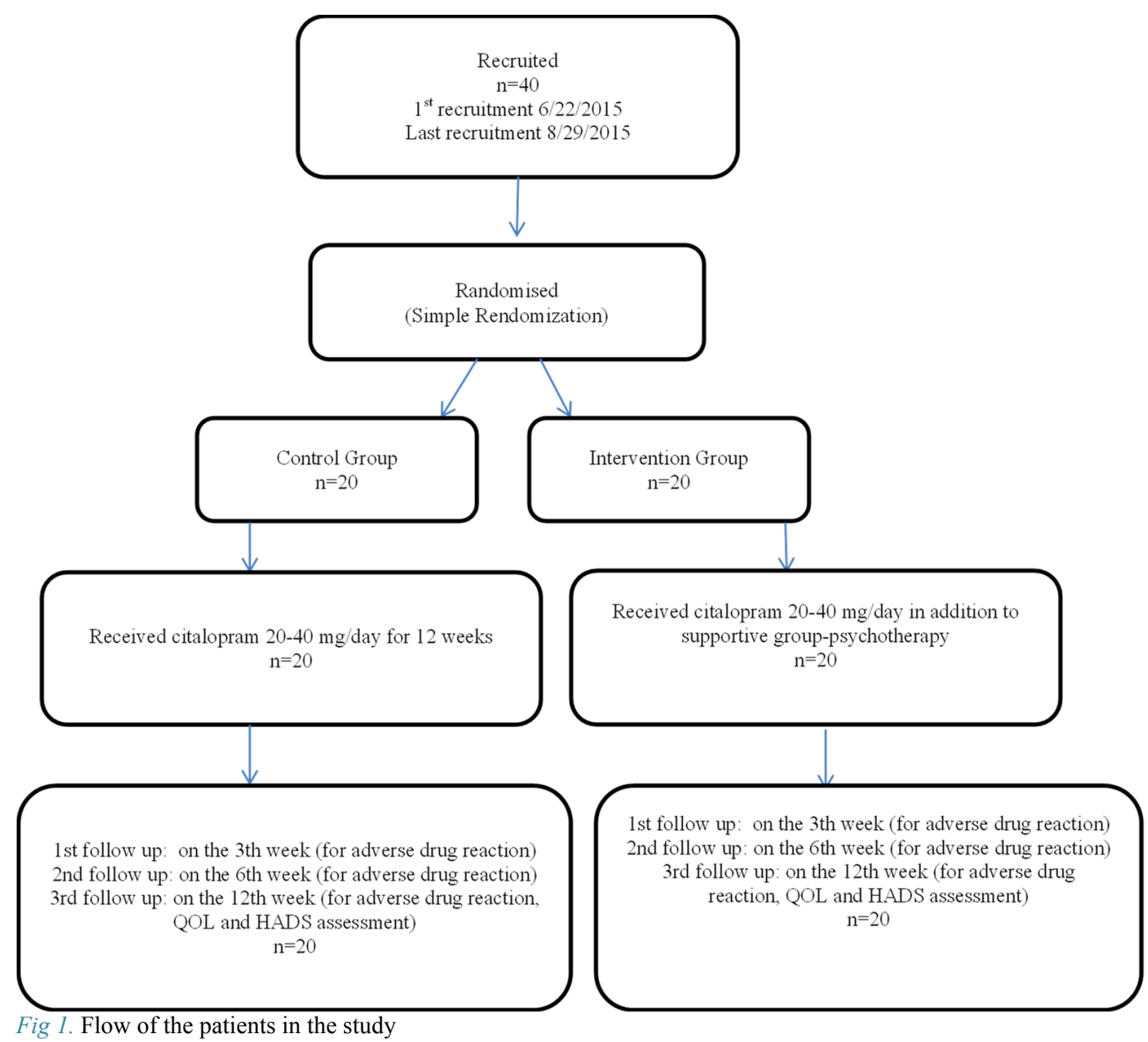

previous class assignment; describing situations, recognizing and expressing excitements, describing self-talk as a relationship between situation and excitement using patients' examples; (6) an overview of the previous class assignment; setting up hot thoughts, introducing evidence techniques, and evaluating existing evidence about hot thoughts; (7) an overview of the previous class assignment; empty chair technique training and team members' demonstrations; and training of the role playing technique; (8) application of the skills learned by participants.

All the participants were examined by a psychiatrist on the $3^{\text {rd }}, 6^{\text {th }}$, and $12^{\text {th }}$ weeks; their adverse drug reactions were followed- up, and HADS and WHO QOL-BREF questionnaires were reassessed at the end of the program $\left(12^{\text {th }}\right.$ week). Therapeutic response was defined as $50 \%$ or more reduction in the symptoms compared to the baseline examination. Fortunately, all the patients completed the study and no one dropped out due to death or noncompliance.

The flow of the participants in the study is presented in Fig. 1.

\section{Ethical approval and consent to participate}

Ethical concerns related to clinical trials were considered in the study design, patient participation, informing, confidentiality, and autonomy. Also, the research was registered in the website of Iranian Registry of Clinical Trials (www.irct.ir), which is a part of the WHO Registry Network and is set up by the Ministry of Health and Med- ical Education (MOHME) of Iran with the IRCT2015063022991N1 identification number.

\section{Consent for publication}

Written informed consent for publication of the patients' clinical details was obtained from the participants.

\section{Availability of data and material}

All data generated or analyzed during this study are included in this article and its supplementary information files.

\section{Statistical analysis}

Demographic characteristics and treatment results were compared between the 2 groups. The results are presented as means $\pm \mathrm{SD}$ for continuous data and as $\mathrm{n}(\%)$ for categorical data. The 2 groups had no differences in baseline characteristics; therefore, it was not necessary to adjust the confounding factors.

Data analysis was performed by t test and ANOVA repeated measure at alpha significance level of 0.05 using SPSS software package (Version 18).

\section{Results}

Participants' age ranged from 29 to 75 years. The demographic and clinical characteristics, comorbid disorders, and psychiatric histories of the 2 groups are presented in Table 1. This table demonstrates that age, the area of residence, educational level, marital status, type of cancer 


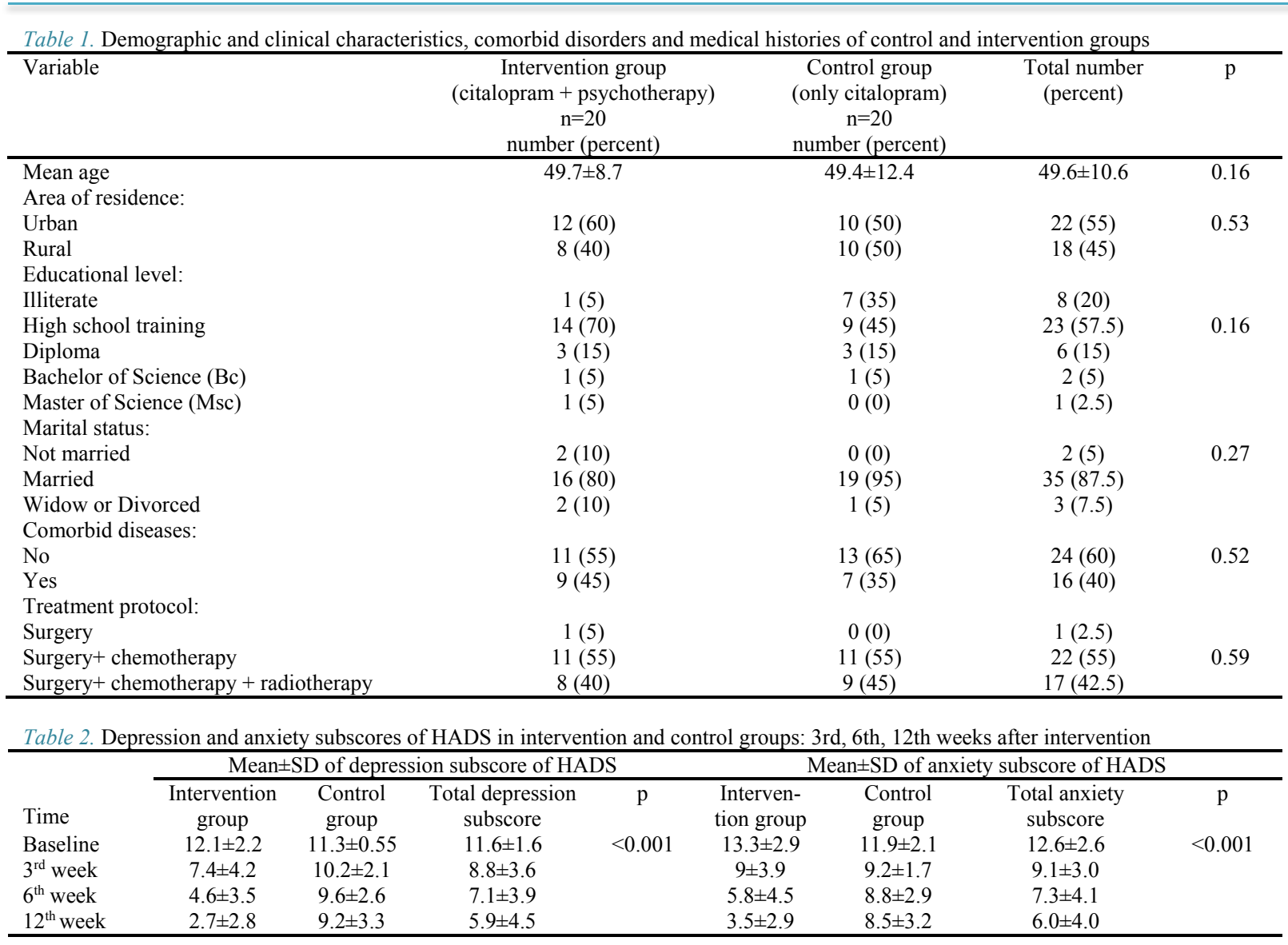

treatment, family history of psychiatric disorders, and comorbid diseases had no significant differences between the 2 groups $(\mathrm{p}>0.05)$. The mean depression subscale and anxiety subscale of HADS were $11.6 \pm 1.6$ and 12.6 \pm 2.6 , respectively, $(95 \% \mathrm{CI}=11.1-12.2$ and $11.8-13.4$, respectively) at baseline examination, which were in the range of symptomatic level of HADS scoring. After the intervention, depression subscales reduced to 7.7-9.8 (on the $3^{\text {rd }}$ week of intervention), 6.1-8.1 (on the $6^{\text {th }}$ week), and 5.0-6.9 (on the $12^{\text {th }}$ week) in the 2 groups. Furthermore, anxiety subscales reduced to 8.1-10.1, 6.1-8.5, and 5.06.9 , respectively. The results showed that depression and

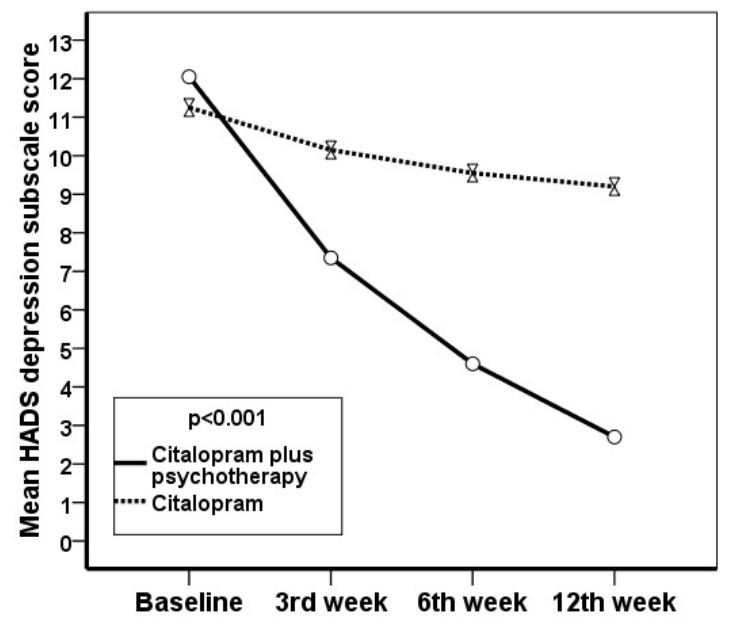

Fig. 2. The trend of depression (based on mean depression subscore of HADS) in the two groups anxiety symptoms of the 2 groups had a significant improvement on the $3^{\text {rd }}$, $6^{\text {th }}$ and $12^{\text {th }}$ weeks of intervention $(\mathrm{p}<0.001)$ and the patients who were treated with citalopram plus group psychotherapy scored in the range without clinical symptoms of anxiety or depression on the $12^{\text {th }}$ week. The scores of depression and anxiety of the 2 groups on the $3^{\text {rd }}, 6^{\text {th, }}$ and $12^{\text {th }}$ weeks of the study are presented in Table 2; and the trend of these scores on the $3^{\text {rd }}$, $6^{\text {th }}$ and $12^{\text {th }}$ weeks of the research are presented in Figs. 2 and 3 .

The occurrence of adverse drug reactions, such as nausea, vomiting, anorexia, sleep disturbance, headache,

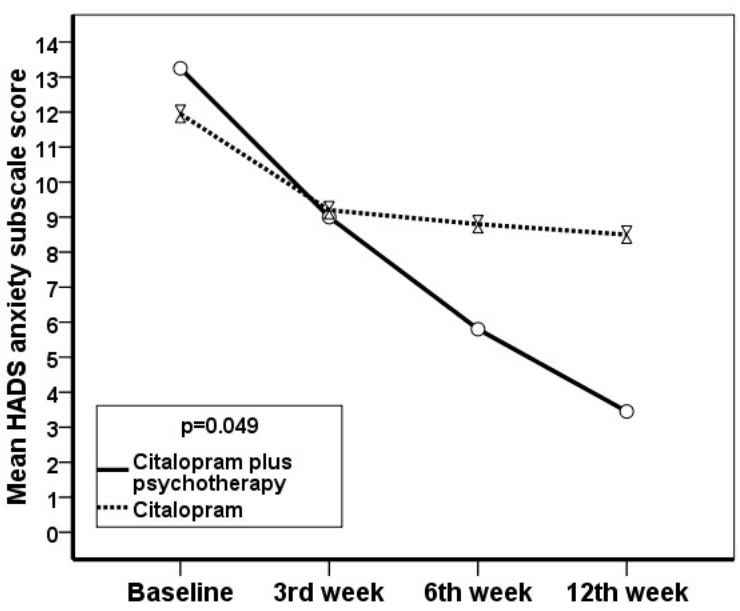

Fig. 3. The trend of anxiety (based on mean anxiety subscore of HADS) in the two groups 
tremor, perspiration, and sexual function impairment were followed in the 2 groups. The findings showed that citalopram did not have any significant adverse effects, also, there was no significant statistical difference between the 2 groups on the incidence of drug side-effects $(p>0.05)$, except for nausea $(p=0.004)$.

The mean standardized score of quality of life (total of 100 points) had no significant difference between the 2 groups before intervention: $44.09 \pm 8.48$ in the case group and $43.64 \pm 13.73$ in control group $(p=0.900)$. After the intervention, QOL measure changed to $81.70 \pm 10.04$ and $47.39 \pm 12.93$, respectively $(\mathrm{p}<0.001)$. This finding shows that the mean score of QOL in the intervention group increased by 1.85 fold, but this score had a slight increase in the control group.

\section{Discussion}

The findings revealed that both depression and anxiety significantly improved in the 2 groups, especially the patients who were treated with citalopram and group psychotherapy were cured completely on the $12^{\text {th }}$ week and had no clinical symptoms. This finding is consistent with the result of Park in Korea (2012) in which a 12-week treatment with escitalopram (5-20 mg/day) in 79 breast cancer patients with depression improved QoL and reduced depression in the participants (1). Moreover, in the research of Vega in Spain, treatment with escitalopram (10-20 mg QD) plus brief narrative therapy in nonmetastatic breast, lung, and colon cancers with depressive disorder made significant improvements in global health and global QoL (21). Fann, in his review article (2008), presented 14 studies in which antidepressants were prescribed for breast cancer patients; in some trials the efficacy of selective serotonin reuptake inhibitors such as fluoxetine, paroxetine, and sertraline has been emphasized; in addition, the effectiveness of psychotherapy approaches on pain, fatigue, and distress reduction, and improvement in depression, anxiety, and QoL have been mentioned (2).

Previous studies suggest that in palliative care of cancer patients, depression is frequently undiagnosed or untreated and that interventions are often initiated so late that patients die before they have time to feel the effect (14). Four common causes of ineffective management of depression in cancer patients are under detection, inadequate initial treatment, failure to monitor their adherence and response to therapeutic approaches, and failure to adjust treatment protocols in non-responding patients (22). There is a bidirectional relationship between depression and chronic diseases such as cancer. The psychobiological changes and adverse health risk behaviors associated with depression can deteriorate cancer prognosis, and biological changes and complications associated with cancer and cancer treatment approaches may precipitate depressive episodes (23). Comorbid depression is associated with increased medical symptom, impairment in global functioning, medical expenditures, lower adherence to selfcare approaches, and increased risk of morbidity and mortality in cancer patients. Depression may worsen the course of cancer because of its effect on hypothalamicpituitary axis, autonomic nervous system, proinflammato- ry factors, and metabolic factors in addition to being associated with a higher risk of a sedentary lifestyle, obesity, smoking, and lower adherence to medical regimens (5). These options emphasize the proper and timely treatment of depression in cancer patients.

In this study, both groups were treated with citalopram, which is one of the most specific SSRIs and its plasma concentration rises in direct proportion to dose; thus, this drug may be a better choice for older adults and patients with significant liver or kidney dysfunction. Additionally, this drug does not substantially inhibit P450 enzymes, therefore, has the least drug interaction with other medications, such as chemotherapy drug regimens (24). In our research, treatment response was observed after the third week, which is compatible with this notion that SSRIs are often described as having a delayed onset of effect in the treatment of depression (25).

Combined therapy had a significant effect on QOL improvement of the patients. This finding is consistent with the result of Kissane's research in which supportiveexpressive group therapy improved quality of life, including treatment of and protection against depression (13). Considering the effects of cancer treatment approaches, such as chemotherapy on different domains of patients' QOL (physical, emotional, mental, and social functioning) (26), psychotherapy was accompanied by training for some important skills, such as problem-solving and relaxation techniques, which can improve patients' QOL.

In this research, sexual dysfunction was reported in $40 \%$ of the patients; this side-effect is one of the most common adverse reactions of citalopram; also, in previously published papers, the frequency of this reaction in the recipients of SSRI was reported to be $50 \%-80 \%$ (27). Other common reactions were nausea and vomiting, which were reported in $17(42.5 \%)$ and $12(30 \%)$ individuals, respectively. Since the patients were undergoing chemotherapy, it was difficult to differentiate nausea and vomiting (N/V) as citalopram side-effects from understandable N/V relating to chemotherapy.

The strength of the present research was that only the breast cancer patients who were at stage III of the disease were enrolled, while in the research of Vega (21), breast, lung, and colon cancer patients were compared with each other. Taking into consideration the different survival of these malignancies, the method of our research has higher priority.

After the treatment, we followed- up the patients only for 12 weeks. Moreover, this particular study design was selected because of the difficulty in setting regular scheduled follow-ups for the recruited cancer patients, especially when they were anxious or depressed. Therefore, we could not evaluate the impact of the intervention on the long survival of the patients. For future studies, it is suggested to implement clinical trials with a much larger sample size and longer follow-up of the patients to assess patients' survival rate.

The challenge of this study was the concurrency of chemotherapy-induced nausea and vomiting, which caused interference in group psychotherapy programs; also, the patients whose homes were far from the interven- 
tion site had to be accommodated with transportation. Moreover, we supported all patients in the 2 groups to help them complete the process of the study.

Considering the effectiveness of early detection of depressive disorders in breast cancer patients, for later sufficient interventions, the first-level health care providers, especially family physicians and health staff, should be trained about the diagnostic criteria of depressive disorders to properly manage those patients who are suspected of having depression by referring them to second-level health care providers, such as psychiatrists, as soon as possible.

\section{Conclusion}

This study showed that combined treatment with citalopram and group psychotherapy has a significant clinical impact on depression, anxiety, and QoL of breast cancer patients without serious physical adverse reactions.

\section{Acknowledgements}

Hereby, we acknowledge the patients who participated in the study.

\section{Conflict of Interests}

The authors declare that they have no competing interests.

\section{References}

1. Park HY, Lee BJ, Kim JH, Bae JN, Hahm BJ. Rapid improvement of depression and quality of life with escitalopram treatment in outpatients with breast cancer: A 12-week, open-label prospective trial. Prog Neuropsychopharmacol Biol Psychiatry. 2012; 36: 318-323.

2. Fann JR, Thomas-Rich AM, Katon WJ, Cowley D, Pepping M, McGregor BA, Gralow J. Major depression after breast cancer: a review of epidemiology and treatment. General Hospital Psychiatr. 2008; 30: $112-126$

3. Deshields T, Tibbs T, Fan MY, Taylor M. Differences in patterns of depression after treatment for breast cancer. Psycho-Oncol. 2006; 15: 398-406.

4. Hjerl K, Andersen EW, Keiding N, Mouridsen HT, Mortensen PB, Jorgensen T. Depression as a prognostic factor for breast cancer mortality. Psychosomatics. 2003; 44: 24-30.

5. Katon WJ. Epidemiology and treatment of depression in patients with chronic medical illness. Dialog Clin Neurosci. 2011;13(1):7-23.

6. Strong V, Waters R, Hibberd C, Murray G, Wall L, Walker J, McHugh G, Walker A, Sharpe M. Management of depression for people with cancer (SMaRT oncology 1): a randomised trial. Lancet. 2008; 372(9632): 40-8.

7. Carvalho AF, Hyphantis T, Sales PM, Soeiro-de-Souza MG, Macêdo DS, Cha DS, McIntyre RS, Pavlidis N. Major depressive disorder in breast cancer: a critical systematic review of pharmacological and psychotherapeutic clinical trials. Cancer Treat Rev. 2014;40:349-355.

8. Ostuzzi G, Benda L, Costa E, Barbui C. Efficacy and acceptability of antidepressants on the continuum of depressive experiences in patients with cancer: Systematic review and meta-analysis. Cancer Treat Rev. 2015; 41(8): 714-724

9. Caraci F, Crupi R, Drago F, Spina E. Metabolic drug interactions between antidepressants and anticancer drugs: focus on selective serotonin reuptake inhibitors and hypericum extract. Curr Drug Metab. 2011; 12(6): 570-7

10. Ashbury JE, Lévesque LE, Beck PA, Aronson KJ. Selective Serotonin Reuptake Inhibitor (SSRI) Antidepressants, Prolactin and Breast Cancer. Front Oncol. 2012; 2: 177.

11. Apler A. Citalopram for major depressive disorder in adults: a systematic review and meta-analysis of published placebo-controlled trials. BMJ Open. 2011; 1(2): e000106.

12. Miguel C, Albuquerque E. Drug interaction in psycho-oncology: antidepressants and antineoplastics. Pharmacology. 2011; 88(5-
6):333-9.

13. Kissane DW, Grabsch B, Clarke DM, Smith GC, Love AW, Bloch $\mathrm{S}$, et al. Supportive-expressive group therapy for women with metastatic breast cancer: survival and psychosocial outcome from a randomized controlled trial. Psycho-Oncol. 2007; 16(4): 277-86.

14. Rayner L, Price A, Hotopf M, Higginson I J. Expert opinion on detecting and treating depression in palliative care: A Delphi study. BMC Palliat Care. 2011; 10: 10.

15. Callari A, Mauri M, Miniati M, Mancino M, Bracci G, Dell'Osso L, Greco C. Treatment of depression in patients with breast cancer: a critical review. Tumori. 2013; 99(5):623-33

16. Nikbakhsh N, Moudi S, Abbasian S, Khafri S. Prevalence of depression and anxiety among cancer patients. Caspian J Intern Med. 2014; 5(3): $167-170$

17. Singer S, Kuhnt S, Götze H, Hauss J, Hinz A, Liebmann A, Krauß $\mathrm{O}$, et al. Hospital anxiety and depression scale cutoff scores for cancer patients in acute care. Br J Cancer. 2009; 100(6): 908-912.

18. Montazeri A, Vahdaninia M, Ebrahimi M, Jarvandi S. The Hospital Anxiety and Depression Scale (HADS): translation and validation study of the Iranian version. Health Qual Life Outcomes. 2003; 1:14.

19. Usefy AR, Ghassemi GR, Sarrafzadegan N, Mallik S, Baghaei AM, Rabiei K. Psychometric Properties of the WHOQOL-BREF in an Iranian Adult Sample. Commu Ment Health J. 2010; 46(2):139-47

20. Nedjat S, Montazeri A, Holakouie K, Mohammad K, Majdzadeh R. Psychometric properties of the Iranian interview-administered version of the World Health Organization's Quality of Life Questionnaire (WHOQOL-BREF): A population-based study. BMC Health Serv Res. 2008; 8: 61

21. Rodríguez Vega B, Palao A, Torres G, Hospital A, Benito G, Pérez E, Dieguez M, Castelo B, Bayón C. Combined therapy versus usual care for the treatment of depression in oncologic patients: a randomized controlled trial. Psycho-Oncol. 2011; 20: 943-952.

22. Kroenke K, Theobald D, Norton K, Sanders R, Schlundt S, McCalley S, Harvey P, Iseminger K, Morrison G, Carpenter JS, Stubbs D, Jacks R, Carney-Doebbeling C, Wu J, Tu W. Indiana Cancer Pain and Depression (INCPAD) Trial Design of a Telecare Management Intervention for Cancer-Related Symptoms and Baseline Characteristics of Study Participants. Gen Hosp Psychiat. 2009; 31(3): 240-253.

23. Voinov B, Richie WD, Bailey RK. Depression and Chronic Diseases: It Is Time for a Synergistic Mental Health and Primary Care Approach. Prim Care Companion CNS Disord. 2013;15(2): PCC.12r01468.

24. Marken PA, Munro JS. Selecting a Selective Serotonin Reuptake Inhibitor: Clinically Important Distinguishing Features. Prim Care Companion J Clin Psychiat. 2000;2(6): 205-210.

25. Taylor MJ, Freemantle N, Geddes JR, Bhagwagar Z. Early Onset of Selective Serotonin Reuptake Inhibitor Antidepressant Action, Systematic Review and Meta-analysis. Arch Gen Psychiat. 2006;63(11): 1217-1223.

26. Singh H, Kaur K, Singh Banipal RP, Singh S, Bala R. Quality of Life in Cancer Patients Undergoing Chemotherapy in a Tertiary Care Center in Malwa Region of Punjab. Indian J Palliat Care. 2014;20(2): $116-122$.

27. Sadock BJ, Sadock VA. Kaplan and Sadock's Synopsis of Psychiatry: Behavioral Sciences and Clinical Psychiatry. 11th edition. Philadelphia: Wolters Kluwer. 2015. 